\title{
Imaging the life story of nanotube devices
}

\author{
T. D. Yuzvinsky, W. Mickelson, S. Aloni, S. L. Konsek, A. M. Fennimore, G. E. Begtrup, \\ A. Kis, B. C. Regan, and A. Zettl ${ }^{\text {a) }}$ \\ Department of Physics, University of California at Berkeley, Materials Sciences Division, Lawrence \\ Berkeley National Laboratory, The Molecular Foundry, Materials Sciences Division, Lawrence Berkeley \\ National Laboratory, and Center of Integrated Nanomechanical Systems, Berkeley, California \\ 94720
}

(Received 1 April 2005; accepted 11 July 2005; published online 15 August 2005)

\begin{abstract}
Live imaging of operating multiwall carbon nanotube (MWCNT-) based electronic devices is performed by high resolution transmission electron microscopy. Our measurements allow us to correlate electronic transport with changes in device structure. Surface contamination, contact annealing, and sequential wall removal are observed. Temperature profiles confirm diffusive conduction in MWCNTs in the high bias limit. This technique provides a general platform for studying nanoscale systems, where geometric configuration and electronic transport are intimately connected. () 2005 American Institute of Physics. [DOI: 10.1063/1.2012529]
\end{abstract}

Nanomaterials such as nanotubes, nanowires, nanocrystals, and supramolecular structures have been proposed as the basic building blocks for a new generation of electronic and mechanical systems, including memory and logic components, ${ }^{1-3}$ light-emitting devices and photodetectors, ${ }^{4-6}$ electromechanical actuators, ${ }^{7}$ biological imaging technologies, ${ }^{8}$ and drug delivery systems. ${ }^{9}$ With their small size and high surface-to-volume ratio, nanostructure devices can be faster, cheaper, more efficient, and more sensitive than their conventional analogs.

The same attributes that make nanostructures attractive, however, can also cause undesirable effects. Behavior can be irreproducible and exhibit time dependence or changes in chemical sensitivity from one device to the next without any macroscopic change in fabrication methods or operating environment. Understanding of device variance has been limited by a lack of techniques that can efficiently correlate minute changes in a device's structure with its operational behavior.

Ideally, one would study these correlations by imaging an operating device, in its entirety, with real time, atomic resolution. Transmission electron microscopy (TEM) uniquely satisfies these imaging requirements, but standard device architectures do not allow electron transmission. Silicon nitride $\left(\mathrm{Si}_{3} \mathrm{~N}_{4}\right)$ membranes have been used as electrontransparent supports for TEM imaging. ${ }^{10-12}$ We have adapted this technique to construct electron-transparent devices that can be operated inside a TEM.

Figure 1 shows several such devices. Details of the device fabrication will be presented elsewhere, but briefly, 500-800 nm of silicon oxide is grown on a silicon wafer, after which $10-20 \mathrm{~nm}$ of silicon nitride is deposited. The silicon is then selectively back-etched with $\mathrm{KOH}$. The oxide and nitride layers are exposed to $\mathrm{HF}$, which removes silicon oxide and leaves the silicon nitride intact. Nanostructures are placed on the resulting membrane and located with scanning electron microscopy (SEM). Contacts to the nanostructures are patterned by electron beam lithography and deposited via the electron-beam evaporation of gold.

\footnotetext{
${ }^{\text {a) }}$ Electronic mail: azettl@physics.berkeley.edu
}

This device architecture provides a framework for the study of different response functions (magnetic, electronic, mechanical, chemical) of a wide variety of nanostructures. As a demonstration, we study the electronic transport of individual multiwall carbon nanotubes (MWCNTs). Despite the many studies of MWCNT devices, ${ }^{13-19}$ there are important outstanding questions regarding the mode of electronic transport, the radial distribution of the current density, and the relationship between this distribution and the failure modes of MWCNTs.

Figure 2 follows a representative MWCNT device through the entire sequence of testing. As fabricated [Fig. $2(\mathrm{a})]$, the device is decorated with gold nanoparticles. The nanoparticle coverage on the surrounding continuous membrane serves as a useful temperature diagnostic.

Device operation begins in the low-bias regime $(<200 \mathrm{mV})$, which produces no apparent structural modification on the short time scale of this experiment. In this limit, our devices typically exhibit a linear current-voltage (I-V) relationship, with resistances on the order of $10 \mathrm{k} \Omega$. As

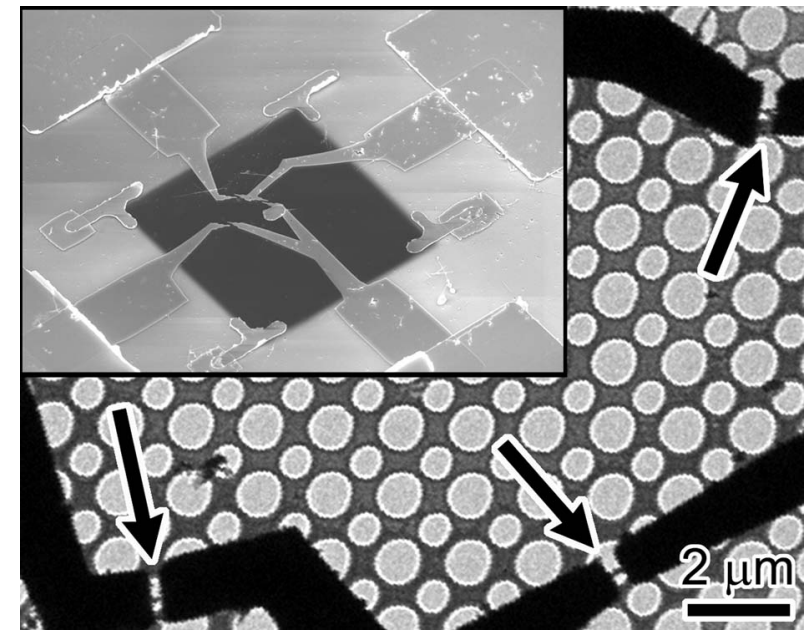

FIG. 1. A TEM image of a membrane with three MWCNT devices indicated by arrows. A regular array of holes is pre-etched into the membrane to allow higher resolution imaging. The scale bar is $2 \mu \mathrm{m}$. Inset: A SEM image of a membrane device with several MWCNTs contacted by gold electrodes. The membrane itself is not visible in the SEM and appears black. 


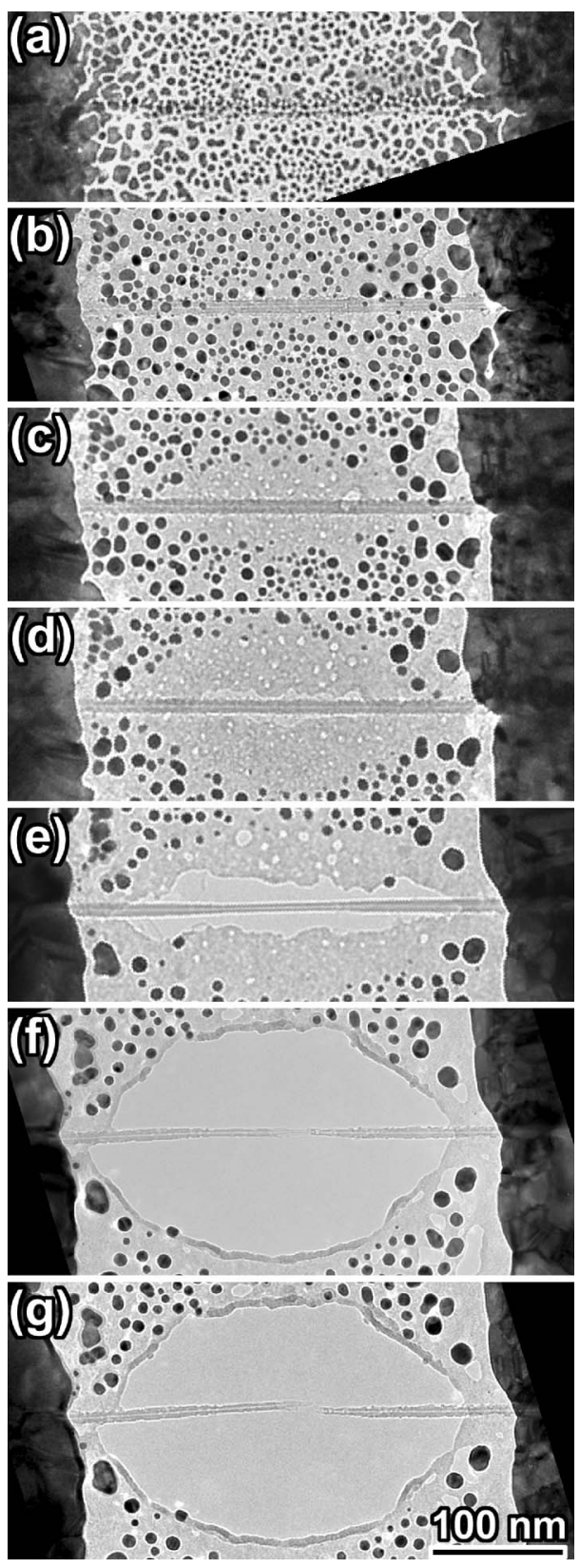

FIG. 2. A series of TEM images showing the evolution of a MWCNT device over time. (a) Gold nanoparticles cover the as-fabricated device. (b) The device is partially cleaned by the application of $1.7 \mathrm{~V}(\sim 190 \mu \mathrm{A})$. (c) Increasing the voltage to $1.72 \mathrm{~V}$ cleans the device further. The $\mathrm{Si}_{3} \mathrm{~N}_{4}$ membrane is beginning to deteriorate. (d) Raising the voltage to $1.9 \mathrm{~V}$ cleans the device of all gold nanoparticles. The membrane under the center section of the MWCNT is gone. (e) The MWCNT has undergone wall-by-wall breakdown and five walls have been removed from the center section. (f) Further breakdown removes all but two complete walls and one partial wall from the center of the MWCNT. (g) The final walls have failed and the MWCNT is now broken into two sections. The scale bar is $100 \mathrm{~nm}$.

the voltage is increased, however, nonlinearities start to appear in the I-V, and at approximately $1 \mathrm{~V}$ the contact edges smooth and recede, with a corresponding increase in resistance. We continue to raise the applied voltage and observe cleaning of the MWCNT, as seen in Fig. 2(b). Heat dissipation in the MWCNT causes nearby gold nanoparticles to evaporate, while nanoparticles further away coalesce into larger particles. Annealing of the contacts begins shortly thereafter and is accompanied by a reduction in the resistance of the device. Both contacts become smoother and the grain size approximately doubles, as seen in Fig. 2(c). ApDownloaded 17 Aug 2005 to 128.32.212.20. Redistribution subject plying current sufficient to anneal the contacts and clean off surface contamination can change the total device resistance without modifying the nanostructure itself.

To simulate prolonged device operation while avoiding excessive beam damage, we continue to increase the input power, which results in localized disintegration of the silicon nitride membrane. Figure 2(d) shows a hole forming beneath the center of the MWCNT. Where the substrate is absent, images of the MWCNTs can be obtained with higher resolution. Suspending nanostructures also eliminates coupling to the substrate during transport measurements.

The evaporation of nanoparticles and the decomposition of the membrane reveal a temperature distribution that peaks midway between the contacts. From the melting point of gold nanoparticles, ${ }^{20}$ we estimate that by Fig. 2(d) the MWCNT has reached temperature in excess of $1200 \mathrm{~K}$, and yet it still shows no damage and continues to function as an effective conductor. The location of the temperature peak clearly indicates that the MWCNT is a diffusive conductor.

Further increasing the voltage drives the MWCNT into current saturation and initiates failure of the MWCNT. As seen in Figs. 2(e) and 2(f), the MWCNT first becomes thinner, with a corresponding discrete resistance increase (to be discussed in greater detail later). Decreasing the applied voltage interrupts the failure process, allowing time for the acquisition of high magnification images. If the process is allowed to continue, the MWCNT ultimately fails [Fig. 2(g)].

The electrically driven thinning of MWCNTs seen in Fig. 2 was first observed in TEM studies of bare MWCNTs. ${ }^{21}$ This phenomenon has been explored both for its physical implications ${ }^{18-22}$ and as a method to modify MWCNTs for use in nanoelectromechanical systems (NEMS). ${ }^{23,24}$ Upon first observation in planar devices ${ }^{17}$ it was attributed to the sequential removal of individual walls, due to the apparent correlation with discrete, equal decreases in current. The previous reports, however, used intrinsically limited imaging methods (SEM, atomic force microscopy) and could not unambiguously correlate each current step to the removal of an individual wall.

Figure 3(a) details the time development of the electronic transport corresponding to the thinning effect seen in Figs. 2(d) and 2(e). From time $t=0$, the voltage is slowly increased in $10 \mathrm{mV}$ steps to $2.56 \mathrm{~V}$. A discrete step in the current response occurs at $2.55 \mathrm{~V}$, followed by four more at $2.56 \mathrm{~V}$. The voltage is then decreased, and the current decreases proportionally. The first current step, from $213.5 \mu \mathrm{A}$, is $\sim 25 \%$ smaller than the following four $(\sim 13.5 \mu \mathrm{A})$. Live imaging during this time period showed five discrete thinning events of the MWCNT, each simultaneous with a step down in current. Figure 3(b) is a high-resolution image taken immediately after the steps were observed, showing that the five outermost walls have been removed from the MWCNT. These data give clear indication of discrete wall-by-wall failure, in which each current step corresponds exactly to the removal of the outermost intact wall.

The mechanism by which these walls are removed is uncertain. Studies $^{16-22}$ of similar devices in ambient atmosphere have attributed wall removal to oxidation. However, the failure documented here occurs in high vacuum, where oxidation is unlikely to play a significant role. Furthermore, Joule heating of MWCNTs essentially halts oxidation in similar vacuum conditions. ${ }^{25}$ Consequently, in the absence of air, an alternate mechanism must be responsible for wall re-
to AlP license or copyright, see http://apl.aip.org/apl/copyright.jsp 

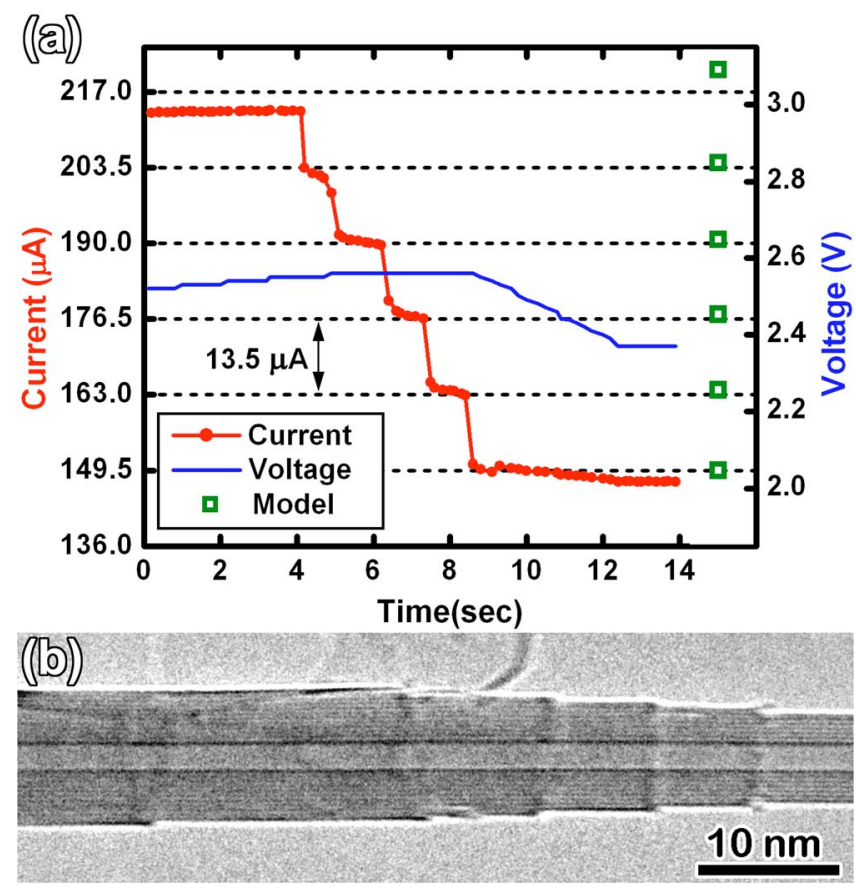

FIG. 3. (Color online) Wall-by-wall breakdown of a MWCNT. (a) The current decreases in a stepwise fashion with remarkably equal current steps of approximately $13.5 \mu \mathrm{A}$. Current steps calculated from a geometric model are shown on the right side of the plot. (b) A TEM image of the MWCNT shows the loss of five walls. The scale bar is $10 \mathrm{~nm}$.

moval. Electron backscattering in nanotubes at high bias generates optical and zone-boundary phonons,${ }^{26}$ which may lead to structural failure in the high current limit.

Our ability to correlate electronic transport with highresolution imaging allows for a quantitative examination of competing models of MWCNT transport. In previous studies of thinning in MWCNT devices, the imaging was performed after the fact and only determined the external dimensions of the MWCNT. The internal structure of the nanotube, including the core size and number of walls, could not be determined. We directly observe how many walls are removed, when they are removed, and over what length.

One model ${ }^{17}$ attributes the current steps to the wall-bywall failure of a saturated MWCNT, and posits that each wall carries equal current. This implies proportionality between current and the number of remaining walls (unmeasured in Ref. 17). Figure 3(b) shows five walls removed from a total of 12. Extrapolating the observed current staircase for seven more steps from $\sim 150 \mu \mathrm{A}$, this model predicts a current of $\sim 50 \mu \mathrm{A}$, even after all the walls have been destroyed.

Another model for MWCNT conduction is one in which current is carried solely by the outer wall, as was reported in measurements of the Aharonov-Bohm effect in MWCNTs at low temperatures. ${ }^{15}$ Adapting this model, which was developed for the low bias limit, to our case, we assume that as each wall fails, conduction passes to the outermost intact wall. To explain the equal current steps, we assume that the current carrying capacity of each wall is linearly proportional to its circumference. For an outer diameter of $9.5 \mathrm{~nm}$ (as measured from TEM images) and the measured initial current of $213.5 \mu \mathrm{A}$, this model predicts current steps of 15.3 $\mu \mathrm{A}$, which is substantially higher than the measured value of $13.5 \mu \mathrm{A}$. From the examination of these two models, we conclude that under these operating conditions, the conduction through the MWCNT is neither solely in the outermost Downloaded 17 Aug 2005 to 128.32.212.20. Redistribution subjec wall, nor is it equally divided among the walls.

Analyzing the MWCNT as if it were a tube of bulk material with a hollow inner core gives competitive agreement with the data. Using the high resolution images, we have measured the MWCNT geometry and calculated the expected resistance, assuming an isotropic conductivity tensor. The material's resistivity $\left(\sim 1.9 \times 10^{-6} \Omega \mathrm{m}\right)$ is calculated from the device's final resistance and geometry. We allow for one free parameter, the contact resistance $(2.2 \mathrm{k} \Omega$ ). Surprisingly, this simple model fits the data rather well, as shown in Fig. 3(a). The singular exception is the first current step, but this step is anomalously small according to all three of the models considered here. The reduced current carrying capacity of the original outer wall may be attributable to damage by TEM beam exposure or surface contaminants.

The authors thank John Cumings for technical assistance during the early stages of this work. This work was supported in part by the Director, Office of Energy Research, Office of Basic Energy Sciences, Materials Sciences Division of the U.S. Department of Energy under Contract No. DE-AC03-76SF00098, and by the National Science Foundation under Grant No. EEC-0425914.

${ }^{1}$ T. Rueckes, K. Kim, E. Joselevich, G. Y. Tseng, C. L. Cheung, and C. M. Lieber, Science 289, 94 (2000).

${ }^{2}$ A. Bachtold, P. Hadley, T. Nakanishi, and C. Dekker, Science 294, 1317 (2001).

${ }^{3}$ S. J. Tans, A. R. M. Verschueren, and C. Dekker, Nature (London) 393, 49 (1998).

${ }^{4}$ M. H. Huang, S. Mao, H. Feick, H. Q. Yan, Y. Y. Wu, H. Kind, E. Weber, R. Russo, and P. D. Yang, Science 292, 1897 (2001).

${ }^{5}$ J. A. Misewich, R. Martel, P. Avouris, J. C. Tsang, S. Heinze, and J. Tersoff, Science 300, 783 (2003).

${ }^{6}$ J. F. Wang, M. S. Gudiksen, X. F. Duan, Y. Cui, and C. M. Lieber, Science 293, 1455 (2001).

${ }^{7}$ A. M. Fennimore, T. D. Yuzvinsky, W. Q. Han, M. S. Fuhrer, J. Cumings, and A. Zettl, Nature (London) 424, 408 (2003).

${ }^{8}$ M. Bruchez, M. Moronne, P. Gin, S. Weiss, and A. P. Alivisatos, Science 281, 2013 (1998).

${ }^{9}$ S. R. Sershen, S. L. Westcott, N. J. Halas, and J. L. West, J. Biomed. Mater. Res. 51, 293 (2000).

${ }^{10}$ S. B. Chikkannanavar and D. E. Luzzi, Nano Lett. 5, 151 (2005).

${ }^{11}$ A. Y. Kasumov, Khodos, II, P. M. Ajayan, and C. Colliex, Europhys. Lett. 34, 429 (1996).

${ }^{12}$ A. Kis, G. Csanyi, J. P. Salvetat, T. N. Lee, E. Couteau, A. J. Kulik, W. Benoit, J. Brugger, and L. Forro, Nat. Mater. 3, 153 (2004).

${ }^{13}$ A. Y. Kasumov, H. Bouchiat, B. Reulet, O. Stephan, Khodos, II, Y. B. Gorbatov, and C. Colliex, Europhys. Lett. 43, 89 (1998).

${ }^{14}$ S. Frank, P. Poncharal, Z. L. Wang, and W. A. de Heer, Science 280, 1744 (1998).

${ }^{15}$ A. Bachtold, C. Strunk, J. P. Salvetat, J. M. Bonard, L. Forro, T. Nussbaumer, and C. Schonenberger, Nature (London) 397, 673 (1999).

${ }^{16}$ P. G. Collins, M. S. Arnold, and P. Avouris, Science 292, 706 (2001).

${ }^{17}$ P. G. Collins, M. Hersam, M. Arnold, R. Martel, and P. Avouris, Phys. Rev. Lett. 86, 3128 (2001)

${ }^{18}$ P. G. Collins and P. Avouris, Appl. Phys. A: Mater. Sci. Process. 74, 329 (2002).

${ }^{19}$ B. Bourlon, C. Miko, L. Forro, D. C. Glattli, and A. Bachtold, Phys. Rev. Lett. 93, 176806 (2004).

${ }^{20}$ K. Koga, T. Ikeshoji, and K. Sugawara, Phys. Rev. Lett. 92, 115507 (2004).

${ }^{21}$ J. Cumings, P. G. Collins, and A. Zettl, Nature (London) 406, 586 (2000).

${ }^{22}$ B. Bourlon, D. C. Glattli, B. Placais, J. M. Berroir, C. Miko, L. Forro, and A. Bachtold, Phys. Rev. Lett. 92, 026804 (2004).

${ }^{23}$ B. Bourlon, D. C. Glattli, C. Miko, L. Forro, and A. Bachtold, Nano Lett. 4, 709 (2004).

${ }^{24}$ A. M. Fennimore, T. D. Yuzvinsky, B. C. Regan, and A. Zettl, AIP Conf. Proc. 723, 587 (2004)

${ }^{25}$ T. D. Yuzvinsky, A. M. Fennimore, W. Mickelson, C. Esquivias, and A. Zettl, Appl. Phys. Lett. 86, 053109 (2005).

${ }^{26}$ Z. Yao, C. L. Kane, and C. Dekker, Phys. Rev. Lett. 84, 2941 (2000). 